\title{
Myocardial Alterations in Adrenoreceptors After Ventricular Unloading with a Pulsatile vs a Nonpulsatile Device
}

\author{
Roger J. Bick ${ }^{* 1}$, Brian J. Poindexter ${ }^{1}$, Pippa M. Evans ${ }^{1}$, Courtney Gemmato ${ }^{2}$ and O.H. Frazier ${ }^{2}$ \\ ${ }^{I}$ Department of Pathology and Laboratory Medicine, The University of Texas Medical School at Houston, Houston, \\ Texas, USA \\ ${ }^{2}$ Center for Cardiac Support, Texas Heart Institute at St. Luke's Episcopal Hospital, Houston, Texas, USA
}

\begin{abstract}
We have recently shown that ventricular unloading with an implantable left ventricular assist device (LVAD) leads to improved calcium handling and membrane integrity and redistribution of alpha adrenoreceptors (AARs) and betaadrenoreceptors (BARs). Here, we used fluorescence deconvolution microscopy to examine the effect of LVAD type (pulsatile vs non-pulsatile) on upregulation and redistribution of adrenoreceptors in core biopsy samples of the myocardium before and after the removal LVAD. We noted no major differences between the pulsatile and non-pulsatile groups; however, an individual patient's 'recovery' in adrenoreceptor numbers depended on the pre-LVAD number of receptors. These findings suggest that ventricular unloading is beneficial regardless of LVAD type; however, the degree of repair and recovery may correlate with the patient's level of ventricular dysfunction at implant and the pre-LVAD number of adrenoreceptors (130).
\end{abstract}

Keywords: Heart failure, adrenoreceptors, microscopy, ventricular unloading.

\section{INTRODUCTION}

Alpha-adrenoreceptors (AARs), beta-adrenoreceptors (BARs), and their subtypes are intimately involved in cell signaling [1] and in modulating heart failure [2]. We have recently shown that ventricular unloading with an implantable left ventricular assist device (LVAD) may lead to improvements in calcium handling and membrane integrity, and to redistribution of both AARs and BARs from perivascular to intramyocytic areas $[3,4]$. Improvements in cardiac function after ventricular unloading with all types of LVADs have been documented [5-10] even in patients who require continued LVAD support $[5,9,10]$. Increased myocardial adrenoreceptor content and sensitivity is a major benefit of ventricular unloading [11], resulting in restoration of optimum myocardial contractility. The use of adrenoreceptors as therapeutic targets has not been well studied. Targeting specific receptor subtypes for treatment regimens may be beneficial and may depend on the type of LVAD used. Thus, we investigated the upregulation and redistribution of adrenoreceptors by comparing tissue samples from patients who were supported by either a pulsatile or a non-pulsatile LVAD.

\section{MATERIALS AND METHODOLOGY}

Myocardial AAR and BAR densities and localizations were compared at the time of LVAD insertion and at either LVAD removal or organ transplantation. This investigation was approved by our center's institutional review board, and the patients provided written informed consent. All

*Address correspondence to this author at the Department of Pathology and Laboratory Medicine, University of Texas Medical School at Houston, MSB 2.288, 6431 Fannin Street, Houston, TX 77030, USA; Tel: 713-500-5406; Fax: 713-500-0730; E-mail: Roger.J.Bick@uth.tmc.edu procedures conformed to guidelines dictated by the University of Texas Medical School at Houston and the Texas Heart Institute. A left ventricular myocardial core removed from the apex $(1-1.5-\mathrm{cm}$ wide) for LVAD placement served as the initial biopsy specimen. A second biopsy sample taken at LVAD explant after heart transplantation was obtained near the apex of the left ventricle but away from the suture line used for LVAD implant. Patient characteristics and preoperative hemodynamic measures were similar in the 2 patient groups before LVAD implantation (Table 1). All patients accepted for heart transplantation were in New York Heart Association class-IV heart failure. The nonpulsatile LVAD patients had an average pulmonary capillary wedge pressure (PCWP) of $25 \pm 4.6 \mathrm{mmHg}$, a cardiac index (CI) of $1.67 \pm 0.22$ $\mathrm{L} / \mathrm{min} / \mathrm{m} 2$, and a central venous pressure (CVP) of $12.2 \pm 2.8$ $\mathrm{mmHg}$. The pulsatile LVAD patients had an average PCWP of $20 \pm 3.4 \mathrm{mmHg}$, a CI of $2.08 \pm 0.28 \mathrm{~L} / \mathrm{min} / \mathrm{m} 2$, and a CVP of $8 \pm 3.1 \mathrm{mmHg}$.

\section{Tissue Preparation and Staining}

Core samples of fresh cardiac tissue were embedded in OCT compound (Tissue-Tek, Torrance, California.) and frozen on dry ice. We used a Reichert HistoSTAT cryotome at $4{ }^{\circ} \mathrm{C}$ to obtain sections $(10 \pm 3 \mu \mathrm{m}$-thick), which were placed on poly-L-lysine-coated (Sigma-Aldrich Corp., St. Louis, Missouri) coverslips and soaked in 3.7\% paraformaldehyde (5 minutes at room temperature). Sections were stained with fluorescent-receptor probes $(5 \mathrm{nmol} / \mathrm{L}$ for 30 minutes at $37^{\circ} \mathrm{C}$ ) and placed on a glass slide under 1 drop of Elvanol (DuPont Antifade, Wilmington, Delaware). To localize AARs, we used the Bodipy 558/568-tagged prazosin probe (Invitrogen/Molecular Probes Inc., Carlsbad, California) at a $K \mathrm{~d}$ of $0.13 \mathrm{nmol} / \mathrm{L}$ for prazosin. We 
Table 1. Patient Characteristics Before Left Ventricular Assist Device Implantation

\begin{tabular}{|l|c|c|}
\hline & Non-Pulsatile & Pulsatile \\
\hline \hline Average age & $58.4 \pm 45.75$ & $47.3 \pm 9.481$ \\
\hline Men/Women & $7-2$ & $9-0$ \\
\hline Days on LVAD & $91.9 \pm 48.4$ & $129 \pm 51.2$ \\
\hline Av. Duration HF (years) & $6.72 \pm 5.36$ & $9.7 \pm 6.3$ \\
\hline Transplanted/expired & $6-3$ & $7-2$ \\
\hline Av. Central venous pressure & $12.2 \pm 2.8$ & $8.0 \pm 3.1$ \\
\hline Av. Cardiac index & $1.67 \pm 0.22$ & $2.08 \pm 0.28$ \\
\hline Av. Capillary Wedge pressure & $25 \pm 4.6$ & $20 \pm 3.4$ \\
\hline
\end{tabular}

capillary wedge pressure in $\mathrm{mmHg}$.

monitored nonspecific binding by preincubating sections with nonradioactive prazosin before incubating the sections with the fluorescent probe. For BAR typing, we used the generic tag CGHP12177 as previously described [3]. The probe for actin was Bodipy FL Phallicidin (Molecular Probes, Eugene Oregon; product \# B607; excitation peak $505 \mathrm{~nm}$, emission peak $512 \mathrm{~nm}$, and $\mathrm{Kd}=38 \mathrm{nM}$ ). We stained nuclei with DAPI (Molecular Probes/Invitrogen). Filters were set as follows: DAPI-excitation $360 \mathrm{~nm}$, band-pass 40nm, emission 457nm, band-pass 50nm; FITC-excitation $490 \mathrm{~nm}$, band-pass $20 \mathrm{~nm}$, emission $528 \mathrm{~nm}$, band-pass $38 \mathrm{~nm}$; TxRed-excitation $555 \mathrm{~nm}$, band-pass $28 \mathrm{~nm}$, emission $617 \mathrm{~nm}$, band-pass $73 \mathrm{~nm}$.
A primary antibody (SMA Sigma Monoclonal; diluted 1:100) followed by a secondary antibody was used to probe for smooth-muscle actin (AlexaFluor 647 antibody; Molecular Probes; goat anti-mouse; diluted 1:500) or cardiac actin (BODIPY and Texas Red-tagged phallicidin; Molecular Probes, diluted 1:100). We used DAPI $(0.1 \mathrm{~g} / \mathrm{ml}$; Molecular Probes) to identify nuclei and cell types (fibroblasts, endothelial cells, and myocytes). All images were magnified at $400 \mathrm{X}$. Image acquisition We used an Applied Precision DeltaVision scanning fluorescence microscope (Issaquah, Washington) fitted with an Olympus IX70 microscope (Melville, New York) with deconvolution capabilities. Obtained in a complete pass from tissue bottom to top, the 0.25 - $\mu \mathrm{m}$-thick images were subjected to deconvolution (5 iterations), stacking, and volume-rendition with Imaris software (Bitplane AG, Zurich, Switzerland).

We counted 3 distinct areas of fluorescence per sample to reduce errors in stereology and captured areas of interest as red-green-blue $(\mathrm{RGB})$ files $(90 \times 90 \mu \mathrm{m})$. The number of pixels in a $60 \times 60-\mu \mathrm{m}$ area was used to determine the mean receptor density. We selected fields with the least amount of fibrosis to compare receptor number between sections. Receptor numbers were measured by a Corel (Corel Corp., Ottawa, Canada) and a SigmaScan program (SPSS Inc., Plover, Wisconsin) to minimize errors.

\section{RESULTS}

AARs and BARs were targeted with fluorescent probes in core biopsy samples of myocardium before and after removal of a pulsatile $(n=9)$ or non-pulsatile $(n=9)$ LVAD. Fluorescent deconvolution microscopy was used to determine the effect of LVAD implantation and subsequent
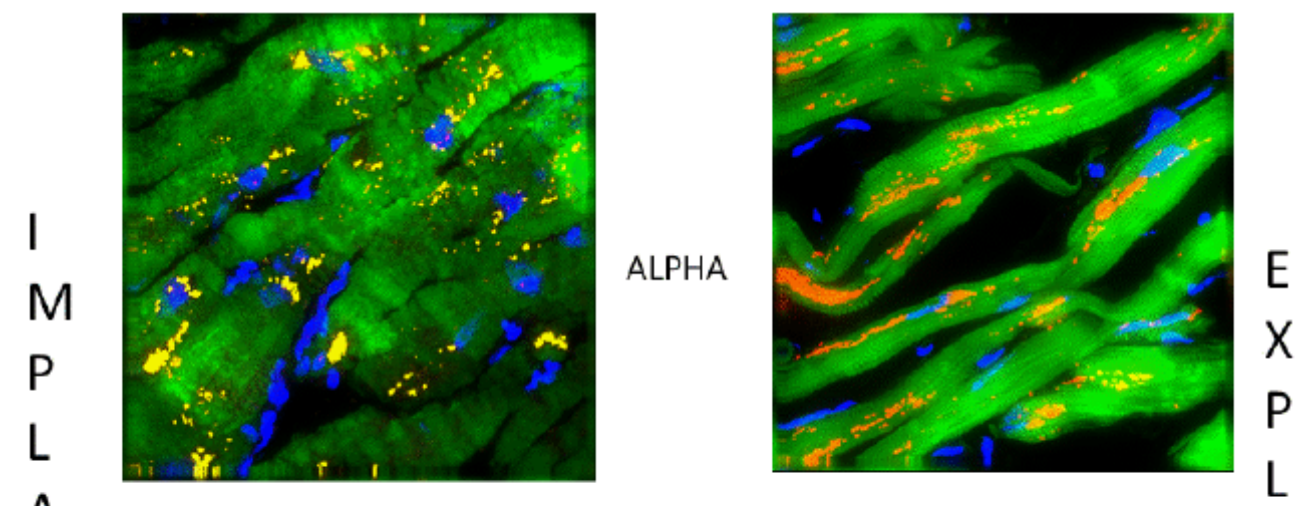

\section{A}
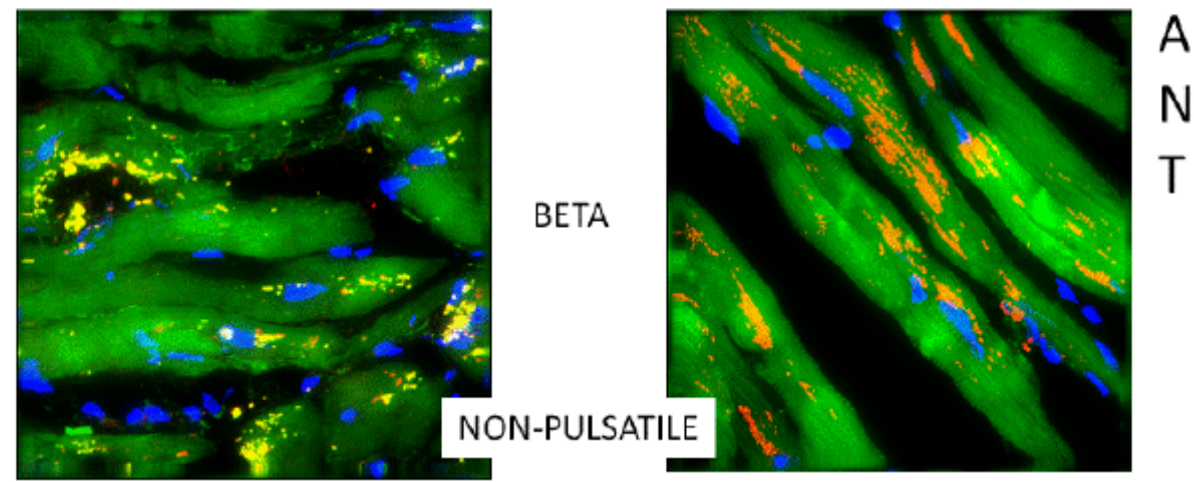

Fig. (1). Alpha-and beta-adrenoreceptor expression before (at implant) and after (at explant) a pulsatile left ventricular assist device was implanted. Green, cardiac actin; blue, nuclei (DAPI); and yellow/red, adrenoreceptors. Magnification 400X. 

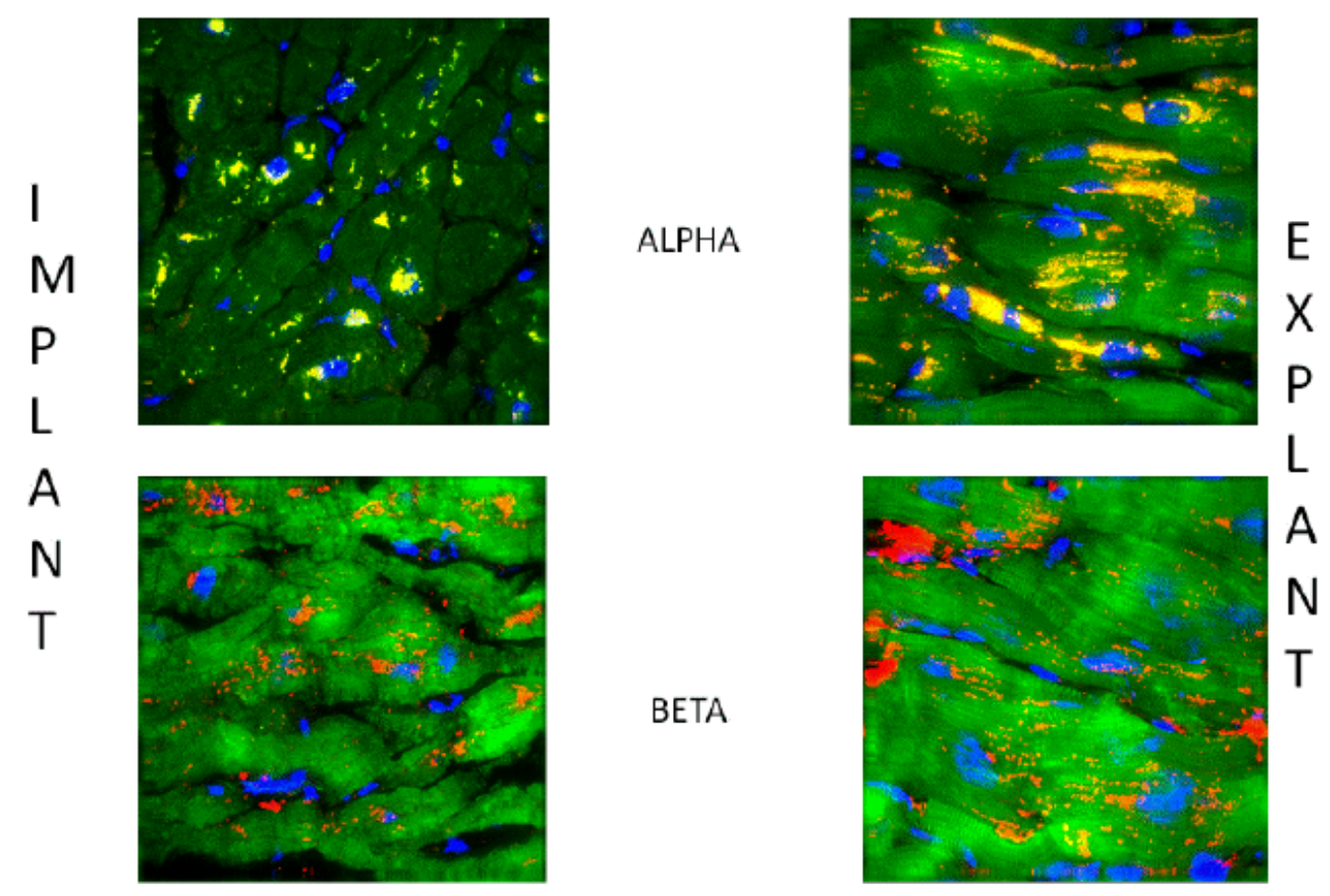

\section{PULSATILE}

Fig. (2). Beta-adrenoreceptor expression before (at implant) and after (at explant) a pulsatile left ventricular assist device was implanted. The number of receptors is not increased by ventricular unloading nor does myocyte structure improve. Green, cardiac actin; blue, nuclei (DAPI); and yellow/red, adrenoreceptors. Magnification 400X.
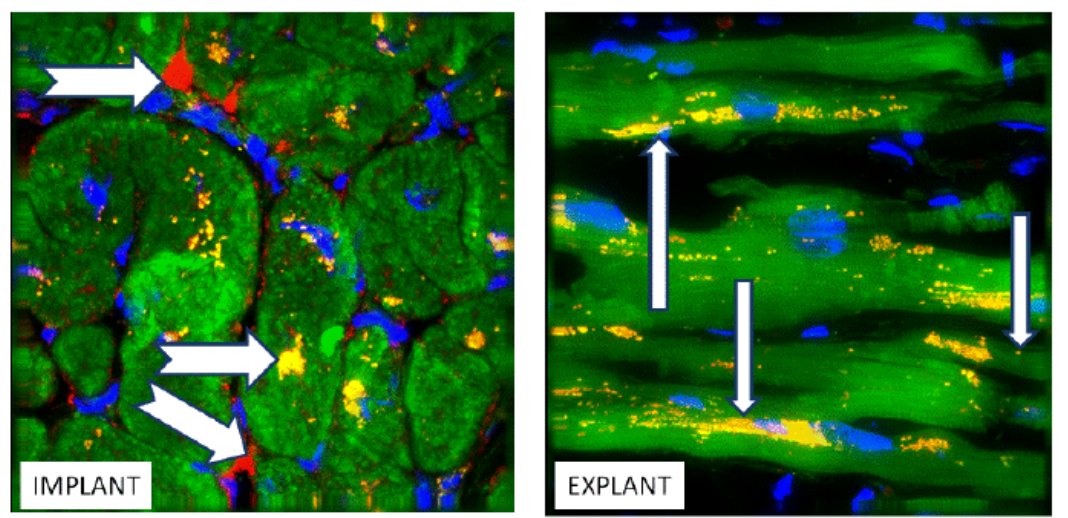

Fig. (3). Alpha-and beta-adrenoreceptor expression before (at implant) and after (at explant) a non-pulsatile left ventricular assist device was implanted. Green, cardiac actin; blue, nuclei (DAPI); and yellow/red, adrenoreceptors. Magnification 400X.

explantation on the density and distribution of adrenoreceptors. The number of BARs decreased from LVAD implant to explant in tissues from patients supported with a pulsatile device (Table 2). These values are somewhat skewed because not all patients had high levels of receptors at the time of device implantation or responded to the LVAD with an increase in receptor number [12]. Figs. $(\mathbf{1}, \mathbf{2})$ present representative implant and explant tissue samples from patients in whom ventricular unloading did not increase the number of BARs, which are localized at the periphery of the myocytes and in the interstitial spaces of the explant (red clusters). In contrast, in these same patients, AARs increased in number after LVAD implantation (Table 2). Fig. (1) shows a tissue sample from a patient with a pulsatile LVAD. At implant, the receptors formed clusters in the intra-fibrillar area (yellow); after implant, these clusters increased in number and became more homogeneously distributed. Fig.
(3) shows a representative tissue sample from a patient with a non-pulsatile LVAD. Both AARs and BARs (yellow/orange-red) increased in number after device implantation (Table 2). In addition, the myofibrillar structure improved at explant, and the AARs and BARs showed a more widespread, intra-fibrillar distribution. Fig. (4) shows another image of the changes in BARs before and after implant of the non-pulsatile device. Both interstitial/perivascular (red) and intra-fibrillar (yellow) clusters of BARs are apparent at implant. At explant, the intra-fibrillar receptors are more widely distributed, and the clusters of interstitial/perivascular receptors are absent. In both patient groups, ventricular unloading caused the adrenoreceptors to increase in number and form a more homogeneous distribution, with the exception of BARs in the pulsatile group. The BARs in this group decreased in number and were mostly distributed in the interstitial spaces. 


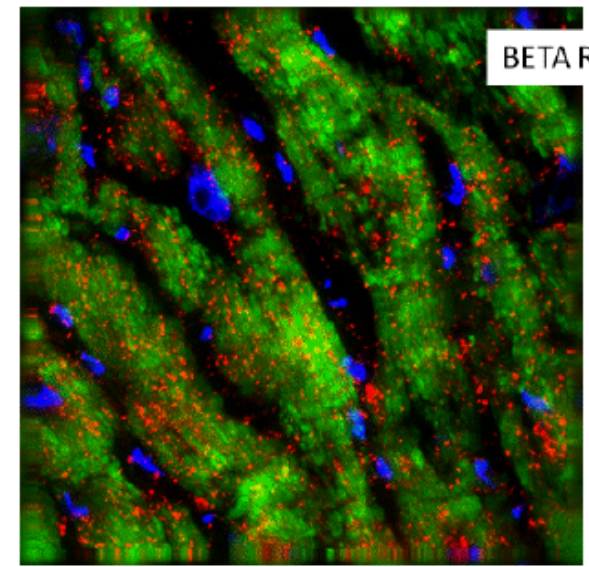

IMPLANT

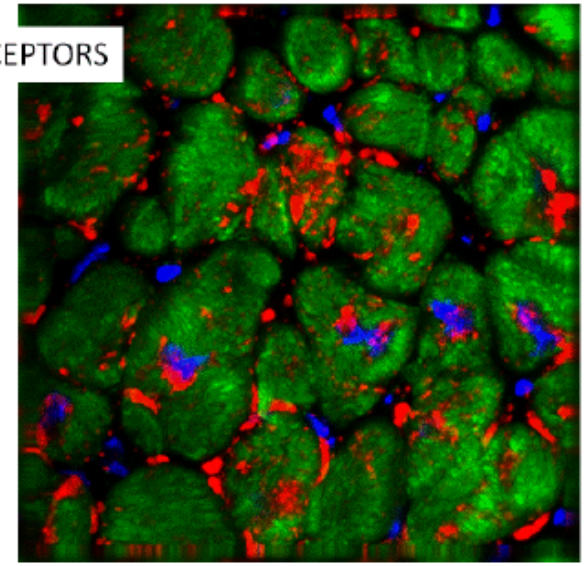

EXPLANT

Fig. (4). Beta-adrenoreceptor expression before (at implant) and after (at explant) a non-pulsatile left ventricular assist device was implanted. Large arrows in the image on the left indicate the clumped and interstitial clusters of beta receptors before ventricular unloading. The arrows on the right indicate the widely distributed clusters of beta-adrenoreceptors after ventricular unloading. Green, cardiac actin; blue, nuclei (DAPI); and yellow/red, adrenoreceptors. Magnification 400X.

Table 2. The Number of Alpha- and Beta-Adrenoreceptors in Patients with Either a non-Pulsatile or Pulsatile Ventricular Unloading Measurements were Made Before (Pre-LVAD) and After (Post-LVAD) the Device was Implanted. AR = Adrenoreceptor

\begin{tabular}{|c|c|c|c|c|}
\hline \multirow{2}{*}{$\begin{array}{c}\text { Adrenoreceptor } \\
\text { Type }\end{array}$} & \multicolumn{2}{|c|}{ Pulsatile } & \multicolumn{2}{c|}{ Non-Pulsatile } \\
\cline { 2 - 5 } & Pre-LVAD & Post-LVAD & Pre-LVAD & Post-LVAD \\
\cline { 2 - 5 } & $\mathbf{N = 9}$ & $\mathbf{N}=\mathbf{9}$ & N=9 & N=9 \\
\hline \hline Beta & $8272 \pm 3042$ & $9751 \pm 4680$ & $8716 \pm 4791$ & $5721 \pm 1475$ \\
\hline Alpha & $5390 \pm 1793$ & $7953 \pm 3225$ & $4656 \pm 1304$ & $7415 \pm 5418$ \\
\hline
\end{tabular}

\section{DISCUSSION}

Our previous studies have shown that LVAD assistance is a viable, long-term treatment option for patients with heart failure. LVADs reportedly reverse cardiac damage, hypertrophy, and fibrosis $[6,13]$, accomplished possibly by restoring AAR and BAR levels, which play an important role in maintaining calcium homeostasis in the heart $[3,4,12]$. Failure to control calcium homeostasis in the heart leads to loss of myocardial contractile function and membrane disruption [4]. Other studies have shown similar results of improvement at the cellular level following ventricular unloading, in a rat model using a beta- 2 antagonist [14], in a multicenter group report [15] and also the "sequestration" of beta-receptors and subsequent recovery in human ventricular samples, similar to our study [16]. We recently studied whether AAR and BAR subtypes undergo similar upregulation and redistribution in human myocardium after implantation of a Jarvik or Thoratec LVAD [12]. Our current objective was to determine whether pulsatile and nonpulsatile ventricular unloading devices differentially affect the number and distribution of AARs and BARs in the heart. Understanding the cellular mechanisms that are initiated during LVAD use is important because particular unloading devices and pharmacologic support may be better suited for specific ailments [17].

This particular collaboration suggests that not only do beta adrenergic receptors become 'dormant' and then respond to ventricular unloading, but that alpha adrenergic receptors respond in a similar manner, if not to such a great extent, possibly initiating a mechanism to avert inotropic changes [18], be part of the repair and restoration process [19], or become a pharmacologic treatment target [20].

Indeed, over 20 years ago alpha-adrenergic targeting drugs were considered as an important part of the physician's arsenal for the treatment of high, blood pressure, hypertension and heart failure [21] and our findings do show extra-myocytic receptors, possibly indicating an association with the microvasculature, but also implicating these proteins in the control of ionic equilibrium and contractionrelaxation well being.

\section{CONCLUSION}

Our studies suggest that ventricular unloading is beneficial regardless of the LVAD type; however, the degree of repair and recovery may correlate with the patient's preLVAD number of AARs and BARs. Results from this study and our previous study indicate that the presence of high numbers of adrenoreceptors at the time of LVAD implantation was predictive of an increase in adrenoreceptor number at explantation [22]. We have also shown that there are a great many variables that lead to a successful recovery and patients often do not respond as expected on the cellular level. This study is limited by the fact that it is observational and descriptive and by the relatively small numbers of patients. In addition, patients who received the non-pulsatile pump were selected based on the strict protocol associated with the investigational device, whereas patients who received the pulsatile pump were chosen based on size. It was also difficult to engage in a 'follow-up' study due to personnel changes, reduced funding and the requirement for the removal of patient identifiers before tissue preparation and fluorescent imaging. Nine percent of the patients were 
explanted in the work group study [15]. The work group study also concluded that "this study has a number of limitations. Because no uniformly accepted criteria exist for device explantation for recovery, criteria for explantation were determined by clinicians at the individual centers and not prescribed in the study protocol. In addition, the observational nature of this study precludes us from ascertaining the recovery rate without LVAD implantation", and these limitations therefore apply to our cellular research. Fortunately, last year a pump was approved by the Federal Drug Administration for permanent placement. (http://www.texasheart.org/AboutUs/News/2010-0121 news_FDAapprove.cfm).

However, these studies suggest that ventricular unloading is beneficial regardless of the type of LVAD; however, the degree of repair and recovery may correlate with the patient's level of ventricular dysfunction at implant and the pre-LVAD number of adrenoreceptors. This work, as do a number of others, strongly suggests that cardiac unloading, by whatever method, is beneficial to the heart failure patient and should be implemented whenever possible. This study also points to alpha-adrenergic receptor involvement in cardiac muscle contraction, a finding that might warrant more consideration regarding heart failure mechanisms, particularly those initiated to allow for later repair and recovery

\section{REFERENCES}

[1] Summers RJ, Molnaar P, Russell F, et al. Coexistence and localization of beta 1 -and beta 2adrenoceptors in the human heart. Eur Heart J 1989; 10 (suppl B): 11-21.

[2] Brodde OE, Zerkowski HR, Doetsch N, et al. Myocardial betaadrenoceptor changes in heart failure: concomitant reduction in beta 1-and beta 2-adrenoceptor function related to the degree of heart failure in patients with mitral valve disease. J Am Coll Cardiol 1989; 14: 323-31.

[3] Bick RJ, Grigore AM, Poindexter BJ, et al. Left ventricular unloading with an assist device results in receptor relocalization as well as increased beta-adrenergic receptor numbers: are these changes indications for outcome? J Card Surg 2005; 20: 332-6.

[4] Grigore A, Poindexter B, Vaughn WK, et al. Alterations in alpha adrenoreceptor density and localization after mechanical left ventricular unloading with the Jarvik flowmaker left ventricular assist device. J Heart Lung Transplant 2005; 24: 609-13.

[5] Ferrari M, Kadipasaoglu KA, Croitoru M, et al. Evaluation of myocardial function in patients with end-stage heart failure during support with the Jarvik 2000 left ventricular assist device. J Heart Lung Transplant 2005; 24: 226-8.
[6] Frazier OH, Benedict CR, Radovancevic B, et al. Improved left ventricular function after chronic left ventricular unloading. Ann Thorac Surg 1996; 62: 675-81.

[7] Hon JK, Yacoub MH. Bridge to recovery with the use of left ventricular assist device and clenbuterol. Ann Thorac Surg 2003; 75: S36-41.

[8] Radovancevic B, Vrtovec B, Frazier OH. Left ventricular assist devices: an alternative to medical therapy for end-stage heart failure. Curr Opin Cardiol 2003; 18: 210-4.

[9] Dandel M, Weng Y, Siniawski H, et al. Long-term results in patients with idiopathic dilated cardiomyopathy after weaning from left ventricular assist devices. Circulation 2005; 112: 137-45.

[10] Frazier OH, Myers TJ, Westaby S, Gregoric ID. Use of the Jarvik 2000 left ventricular assist system as a bridge to heart transplantation or as destination therapy for patients with chronic heart failure. Ann Surg 2003; 237: 631-6; discussion 6-7.

[11] Ogletree-Hughes ML, Stull LB, Sweet WE, et al. Mechanical unloading restores beta-adrenergic responsiveness and reverses receptor down-regulation in the failing human heart. Circulation 2001; 104: 881-6.

[12] Schnee PM, Shah N, Bergheim M, et al. Reversal of myocyte hypertrophy by ventricular unloading: cardiac improvement without adrenergic receptor up-regulation and relocalization. Med Gen Med 2006; 8: 45.

[13] Bick RJ, Poindexter BJ, Buja LM, et al. Improved sarcoplasmic reticulum function after mechanical left ventricular unloading. $\mathrm{J}$ Cardiovasc Pathobiol 1998; 2(3-4): 159-66.

[14] Soppa GK, Lee J, Stagg MA, et al. Role and possible mechanisms of clenbuterol in enhancing reverse remodelling during mechanical unloading in murine heart failure. Cardiovasc Res 2008; 77: 159-66

[15] Maybaum S, Mancini D, Xydas S, et al. Cardiac improvement during mechanical circulatory support: a prospective multicenter study of the LVAD Working Group. Circulation 2007; 115: 2497505.

[16] Perrino C, Schroder JN, Lima B, et al. Dynamic regulation of phosphoinositide 3-kinase-gamma activity and beta-adrenergic receptor trafficking in end-stage human heart failure. Circulation 2007; 116: 2571-9.

[17] Birks EJ, Tansley PD, Hardy J, et al. Left ventricular assist device and drug therapy for the reversal of heart failure. N Engl J Med 2006; 355: 1873-84.

[18] Wang GY, Yeh CC, Jensen BC, et al. Heart failure switches the $\mathrm{RV}$ alpha1-adrenergic inotropic response from negative to positive. Am J Physiol Heart Circ Physiol 2010; 298: H913-30.

[19] Triposkiadis F, Karayannis G, Giamouzis G, et al. The sympathetic nervous system in heart failure physiology, pathophysiology, and clinical implications. J Am Coll Cardiol 2009; 54: 1747-62.

[20] Jensen BC, Swigart PM, De Marco T, et al. \{alpha\} 1-Adrenergic receptor subtypes in nonfailing and failing human myocardium. Circ Heart Fail 2009; 2: 654-63.

[21] Colucci WS. Alpha-adrenergic receptor blockade with praqzosin Consideration of hypertension, heart failure, and potential new applications. Ann Intern Med 1982; 97: 67-77.

[22] Schnee PM, Shah N, Bergheim M. et al. Location and density of alpha and beta adrenoreceptor sub-types in myocardium after mechanical left ventricular unloading. J Heart Lung Transplant 2008; $27: 710-7$.

(C) Bick et al.; Licensee Bentham Open.

This is an open access article licensed under the terms of the Creative Commons Attribution Non-Commercial License (http://creativecommons.org/licenses/by$\mathrm{nc} / 3.0 /$ ) which permits unrestricted, non-commercial use, distribution and reproduction in any medium, provided the work is properly cited. 